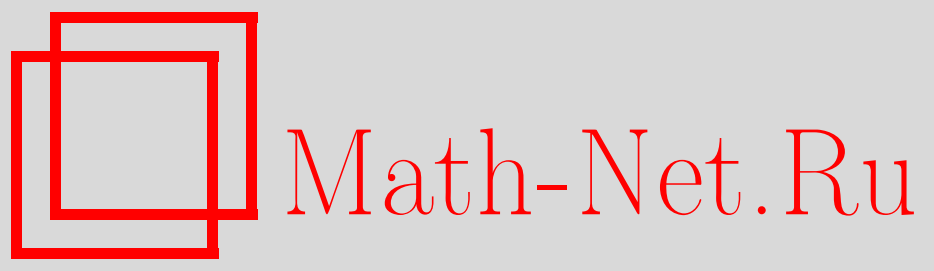

М. А. Башов, С. Н. Селезнева, О длине функций $k$ значной логики в классе полиномиальных нормальных форм по модулю $k$, Дискрет. матем., 2014, том 26, выпуск $3,3-9$

DOI: https://doi.org/10.4213/dm1286

Использование Общероссийского математического портала Math-Net.Ru подразумевает, что вы прочитали и согласны с пользовательским соглашением http: //www . mathnet.ru/rus/agreement

Параметры загрузки:

IP: 35.174 .16 .151

26 апреля 2023 г., 15:31:18

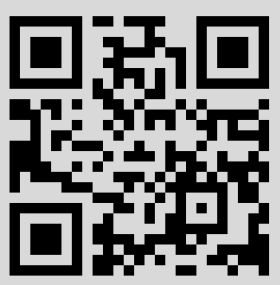




\title{
О длине функций $k$-значной логики в классе полиномиальных нормальных форм по модулю $k$
}

\author{
() 2014 г. М. А. Башов* , С. Н. Селезнева**
}

В работе рассматриваются полиномиальные нормальные формы для функций $k$-значных логик (при простых $k$ ). Полиномиальная нормальная форма по модулю $k$ (п.н.ф.) - это сумма по модулю $k$ произведений переменных или переменных с одним или несколькими отрицаниями Поста, взятых с некоторыми коэффициентами. Длиной п.н.ф. называется число ее различных слагаемых с ненулевыми коэффициентами. Если $k$ - простое число, то каждая функция $k$-значной логики может быть представлена различными п.н.ф. Длиной функции $k$-значной логики в классе п.н.ф. называется минимальная длина среди всех п.н.ф., представляющих эту функцию. Функция Шеннона $L_{k}^{p . n . f .}(n)$ длины функций $k$-значной логики в классе п.н.ф. - это максимальная длина функции в классе п.н.ф. среди всех функций $k$-значной логики, зависящих от $n$ переменных. В работе устанавливается порядок функции Шеннона $L_{k}^{p . n . f}(n)$ (при простых $k): L_{k}^{p . n . f .}(n)=\Theta\left(\frac{k^{n}}{n}\right)$.

Работа поддержана РФФИ, грант 13-01-00958-а и частично грант 13-01-00684-a.

Ключевые слова: функция $k$-значной логики, булева функция, полиномиальное представление, полиномиальная нормальная форма, длина, функция Шеннона, затеняющее множество, покрытие, минимальное покрытие.

\section{1. Введение}

Полиномиальные формы являются одним из способов представления булевых и конечнозначных функций. Полиномиальные представления булевых функций находят применения при синтезе схем, в программируемых логических матрицах (ПЛМ) [1, 2]. Эффективность ПЛМ зависит от числа слагаемых в полиномиальной форме, по которой она построена. В ряде работ изучается сложность представления булевых функций и функций $k$-значной логики полиномиальными формами различных видов $[5,6,7,8]$.

Полиномиальные нормальные формы (п.н.ф.) для булевых функций - это представление булевых функций суммами по модулю два элементарных конъюнкций. Это представление булевых функций аналогично дизъюнктивным нормальным формам (д.н.ф.), только вместо операции дизъюнкции применяется операция сложения

* Место работы: МГУ имени М.В. Ломоносова, e-mail: max.bashov@gmail.com

** Место работы: МГУ имени М.В. Ломоносова, e-mail: selezn@cs.msu.su 
по модулю два. Теоретические и экспериментальные исследования показывают, что представление булевых в виде п.н.ф. более эффективно, чем в виде д.н.ф.: и в худшем случае (при росте числа переменных функций), и для функций малого числа переменных п.н.ф. имеют меньшее число слагаемых, чем соответствующие д.н.ф [2, 7]. Полиномиальные нормальные формы по модулю $k$ для функций $k$-значной логики обобщают понятие п.н.ф. для булевых функций.

В [7] была получена верхняя оценка длины п.н.ф. булевых функций (в худшем случае), в [8] была получена аналогичная верхняя оценка для п.н.ф. функций $k$-значной логики (при простых $k$ ). Значения этих верхних оценок зависят от мощности некоторых определенных комбинаторных множеств. В настоящей работе мы улучшаем оценку из [8] и получаем порядок функции Шеннона длины функций $k$-значной логики в классе п.н.ф. (при простых $k$ ).

\section{2. Основные понятия}

Пусть $k \geqslant 2$ - натуральное число, $E_{k}=\{0,1, \ldots, k-1\}$. Определим на наборах из множества $E_{k}^{n}$ частичный порядок: если $\alpha=\left(a_{1}, \ldots, a_{n}\right) \in E_{k}^{n}$ и $\beta=\left(b_{1}, \ldots, b_{n}\right) \in$ $E_{k}^{n}$, то $\alpha \leqslant \beta$ при $a_{i} \leqslant b_{i} i=1, \ldots, n$. Весом набора $\alpha=\left(a_{1}, \ldots, a_{n}\right) \in E_{k}^{n}$ назовем число $|\alpha|=\sum_{i=1}^{n} a_{i}$ (здесь рассматривается сумма целых чисел). Если $\alpha, \beta \in E_{k}^{n}$, то $\alpha+\beta, \alpha-\beta \in E_{k}^{n}-$ это наборы, полученные соответственно покординатной суммой и разностью по модулю $k$ наборов $\alpha$ и $\beta$.

Для набора $\alpha \in E_{k}^{n}$ назовем его тенъю «вниз» множество

$$
\check{S}(\alpha)=\left\{\beta \in E_{k}^{n}: \beta \leqslant \alpha,|\beta|=|\alpha|-1\right\} .
$$

Заметим, что если $\beta \in \check{S}(\alpha)$, то $|\alpha-\beta|=1$. Если $T \subseteq E_{k}^{n}$, то $\check{S}(T)=\bigcup_{\alpha \in T} \check{S}(\alpha)$.

Множество $T, T \subseteq E_{k}^{n}$, назовем затеняющим на множестве $E_{k}^{n}$, если $\check{S}(T)=$ $E_{k}^{n} \backslash \widehat{(k-1)}$, где $\widetilde{(k-1)}=(k-1, \ldots, k-1)$ - наибольший набор из $E_{k}^{n}$. Множество $T, T \subseteq E_{k}^{n}$, назовем покрытием (асимметричным покрытием «вниз») множества $E_{k}^{n}$, если $T \cup \check{S}(T)=E_{k}^{n}$.

Моном $\prod_{a_{i} \neq 0} x_{i}^{a_{i}}$ назовем соответствующим набору $\alpha=\left(a_{1}, \ldots, a_{n}\right) \in E_{k}^{n}$ и обозначим через $X_{\alpha}$. По определению положим, что константа 1 соответствует набору из всех нулей. Заметим, что если $\alpha, \beta \in E_{k}^{n}$, и $\beta \in \check{S}(\alpha)$, то моном $X_{\alpha-\beta}$ является некоторой переменной.

Функцией $k$-значной логики называется отображение $f^{n}: E_{k}^{n} \rightarrow E_{k}, n=0,1, \ldots$ Множество всех $k$-значных функций обозначим через $P_{k}$, множество всех $k$-значных функций, зависящих от переменных $x_{1}, \ldots, x_{n}$, обозначим через $P_{k}^{n}$. Если $k$ - простое число, то каждая функция $k$-значной логики $f\left(x_{1}, \ldots, x_{n}\right)$ может быть однозначно задана формулой вида

$$
f\left(x_{1}, \ldots, x_{n}\right)=\sum_{\alpha \in E_{k}^{n}: c_{f}(\alpha) \neq 0} c_{f}(\alpha) X_{\alpha},
$$

где $c_{f}(\alpha) \in E_{k}-$ коэффициенты, $\alpha \in E_{k}^{n}$, и операции сложения и умножения рассматриваются по модулю $k$ [9]. Это представление функций $k$-значной логики называется ее полиномом по модулю $k$. При простых значениях числа $k$ однозначно 
определенный полином по модулю $k$ для функции $k$-значной логики $f$ будем обозначать $P(f)$.

Везде в дальнейшем в выражениях для функций $k$-значной логики под знаками + , - , , / / мы будем понимать сложение, вычитание, умножение и деление по модулю $k$ соответственно (при простых $k$ ). В выражениях, не относящихся к функциям $k$-значных логик, под этими знаками будут пониматься соответствующие операции с действительными числами.

Определим полиномиальные нормальные формы по модулю $k$. Под поляризованной переменной $x_{i}$ будем понимать выражение вида $x_{i}+h$, где $h \in E_{k} \backslash\{0\}$. Произведение вида $y_{i_{1}}^{m_{1}} \cdot \ldots \cdot y_{i_{r}}^{m_{r}}$, где $y_{i_{j}}$ есть либо переменная $x_{i_{j}}$, либо поляризованная переменная $x_{i_{j}}, y_{i_{s}} \neq y_{i_{t}}$ при $s \neq t, 1 \leqslant m_{1}, \ldots, m_{r} \leqslant k-1$, назовем мономом с поляризованными переменными. Обычный моном является частным случаем монома с поляризованными переменными.

Выражение вида $\sum_{i=1}^{l} c_{i} \cdot X_{i}$, где $c_{i} \in E_{k} \backslash\{0\}$ - коэффициенты, $X_{i}$ - различные мономы с поляризованными переменными, $i=1, \ldots, l$, назовем полиномиальной нормальной формой по модулю $k$ (п.н.ф.). Длиной п.н.ф. называется число ее слагаемых с ненулевыми коэффициентами. Мы будем полагать константу 0 п.н.ф. с длиной, равной 0 .

Каждую функцию $k$-значной логики (при простых $k$ ) можно представить различными п.н.ф. по модулю $k$. Назовем длиной функции $f$ в классе п.н.ф. величину $l^{p . n . f .}(f)$, равную минимальной длине среди всех п.н.ф., представляющих функцию

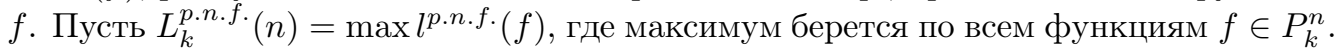

К.Д. Кириченко [7] была исследована длина булевых функций в классе п.н.ф. и получена оценка $L_{2}^{p . n . f \cdot}(n) \leqslant 2 \cdot \frac{2^{n}}{n}\left(\log _{2} n+1\right)$. Эта оценка была получена методом построения п.н.ф. на основе затеняющего множества на $E_{2}^{n}$. С учетом результата из [10] можно получить, что $L_{2}^{p . n . f .}(n)=O\left(\frac{2^{n}}{n}\right)$. В [8] доказана оценка длины $k$-значных функций в классе п.н.ф. по модулю $k$ (при простых $k$ ): $L_{k}^{p . n . f \cdot}(n) \leqslant(2+o(1)) \cdot \frac{k^{n}}{n} \cdot \ln n$. В [8] при построении затеняющего множества на $E_{k}^{n}$ был применен градиентный алгоритм.

В настоящей работе мы обобщаем результат из [10] на множество $E_{k}^{n}$ и получаем порядок функции Шеннона $L_{k}^{p . n . f \cdot}(n)$ при простых $k$.

\section{3. Верхняя оценка сложности функций $k$-значной ло- гики в классе п.н.ф.}

Теорема 1. Пусть $k$ - простое число. Если $T, T \subseteq E_{k}^{n},-$ покрытие множества $E_{k}^{n}$, то для каждой функиии $f\left(x_{1}, \ldots, x_{n}\right) \in P_{k}^{n}$ можно построить п.н.ф. по модулю $k$, ее представляющую, с длиной, не большей $2 \cdot|T|$.

Доказательство. Пусть $T=\left\{\alpha_{1}, \ldots, \alpha_{|T|}\right\}$ - покрытие множества $E_{k}^{n}$, причем $\left|\alpha_{i}\right| \geqslant\left|\alpha_{j}\right|$ при всех $i, j, 1 \leqslant i<j \leqslant|T|$.

Мы опишем алгоритм, в котором последовательно выполняются шаги $1,2, \ldots$, $|T|$. После шага $j$ мы будем получать такие полином $P_{j}$ и п.н.ф. $Q_{j}$, что $f\left(x_{1}, \ldots, x_{n}\right)=Q_{j}+P_{j}$. Кроме того, для каждого слагаемого $X_{\beta}, \beta \in E_{k}^{n}$, с ненулевым коэффициентом в полиноме $P_{j}$ будет верно, что $\beta \notin \bigcup_{i=1}^{j}\left(\left\{\alpha_{i}\right\} \cup \check{S}\left(\alpha_{i}\right)\right)$.

Перед началом работы алгоритма положим $Q_{0}=0, P_{0}=P(f)$. 
LIIa $j$.

1) Пусть $\alpha_{j}=\left(a_{1}, \ldots, a_{n}\right) \in E_{k}^{n}$. Пусть для каждого $\beta \in\left\{\alpha_{j}\right\} \cup \check{S}\left(\alpha_{j}\right)$ моном $X_{\beta}$ входит в полином $P_{j-1}$ с коэффициентом $c_{\beta}$.

Для каждого набора $\beta \in \check{S}\left(\alpha_{j}\right)$ рассмотрим набор $\alpha-\beta \in E_{k}^{n}$. Этот набор имеет вид

$$
\alpha-\beta=(0, \ldots, 0,1,0, \ldots, 0)
$$

Пусть $i_{\beta}, 1 \leqslant i_{\beta} \leqslant n$, номер единственной позиции в наборе $\alpha-\beta$, в которой находится единица. Положим $\check{X}_{\alpha_{j}}=\prod_{\beta \in \check{S}(\alpha)}\left(X_{\alpha-\beta}+\frac{c_{\beta}}{a_{i_{\beta}}}\right)^{a_{i_{\beta}}}$. Заметим, что $\check{X}_{\alpha_{j}}-$ это моном с поляризованными переменными.

2) Пусть $P_{j}$ есть полином, полученный в результате приведения подобных слагаемых в полиномиальном выражении $P_{j-1}+\left(1-c_{\alpha_{j}}\right) X_{\alpha_{j}}-P\left(\check{X}_{\alpha_{j}}\right)$. Пусть $Q_{j}-$ это п.н.ф. $Q_{j-1}+\check{X}_{\alpha_{j}}+\left(c_{\alpha_{j}}-1\right) X_{\alpha_{j}}$.

Заметим, что полином $P\left(\check{X}_{\alpha_{j}}\right)$ содержит слагаемое $X_{\alpha_{j}}$ с коэффицентом 1 и все слагаемые $X_{\beta}$, где $\beta \in \check{S}\left(\alpha_{j}\right)$, с коэффициентами $c_{\beta}$. По построению все они сокращаются в полиноме $P_{j}$. Все другие слагаемые полинома $P\left(\check{X}_{\alpha_{j}}\right)$ имеют вес, не больший $\left|\alpha_{j}\right|-2$. Поэтому в полином $P_{j}$ не могут входить мономы, соответствующие наборам из $\check{S}\left(\alpha_{j}\right)$. Если $X_{\beta}, \beta \in E_{k}^{n}$, является слагаемым с ненулевым коэффициентом в полиноме $P_{j-1}$, то $\beta \notin \bigcup_{i=1}^{j-1}\left(\left\{\alpha_{i}\right\} \cup \check{S}\left(\alpha_{i}\right)\right)$. Так как $\left|\alpha_{i}\right| \geqslant\left|\alpha_{j}\right|$ для всех $i=1, \ldots, j-1$, то если $X_{\beta}, \beta \in E_{k}^{n}$, является слагаемым с ненулевым коэффициентом в полиноме $P_{j}$, то $\beta \notin \bigcup_{i=1}^{j}\left(\left\{\alpha_{i}\right\} \cup \check{S}\left(\alpha_{i}\right)\right)$.

Кроме того

$$
\begin{aligned}
f\left(x_{1}, \ldots, x_{n}\right)= & P_{j-1}+Q_{j-1}=P_{j-1}+Q_{j-1}+ \\
& +\left(\left(1-c_{\alpha_{j}}\right) X_{\alpha_{j}}-P\left(\check{X}_{\alpha_{j}}\right)\right)+\left(\check{X}_{\alpha_{j}}+\left(c_{\alpha_{j}}-1\right) X_{\alpha_{j}}\right)= \\
= & P_{j}+Q_{j} .
\end{aligned}
$$

3) Теперь если $j<|T|$, то переходим к шагу $j+1$; если $j=|T|$, то алгоритм завершает работу.

Если моном $X_{\beta}, \beta \in E_{k}^{n}$, в полиноме $P_{|T|}$ является слагаемым с ненулевым коэффициентом, то $\beta \notin \bigcup_{i=1}^{|T|}\left(\left\{\alpha_{i}\right\} \cup \check{S}\left(\alpha_{i}\right)\right)$. Но множество $T$ является покрытием множества $E_{k}^{n}$. Значит, полином $P_{|T|}$ не содержит слагаемых с ненулевыми коэффициентами, т.е. $P_{|T|}=0$. Поэтому

$$
f\left(x_{1}, \ldots, x_{n}\right)=P_{|T|}+Q_{|T|}=Q_{|T|}=\sum_{j=1}^{|T|}\left(c_{j} X_{\alpha_{j}}+d_{j} \check{X}_{\alpha_{j}}\right),
$$

где $c_{1}, \ldots, c_{|T|}, d_{1}, \ldots, d_{|T|} \in E_{k}$ - некоторые коэффициенты.

Таким образом мы нашли некоторую п.н.ф., представляющую функцию $f\left(x_{1}, \ldots, x_{n}\right)$, и длина этой п.н.ф. не превосходит $2 \cdot|T|$.

Теорема доказана. 


\section{4. Оценка мощности асимметричного покрытия «ВНИЗ»}

Теперь оценим мощность покрытия множества $E_{k}^{n}$ с помощью конструкции, обобщающей метод доказательства [10] верхней оценки мощности когда, асимметрически покрывающего булев куб.

Обозначим через $w(\alpha)$ количество элементов набора $\alpha \in E_{k}^{n}$, не равных $k-1$. Отметим, что число $w(\alpha)$ равно количеству наборов, в тень «вниз» которых входит набор $\alpha$.

Пусть $T \subseteq E_{k}^{n}-$ произвольное множество наборов. Будем говорить, что множество наборов $T$ покрывает множество $T^{\prime}, T^{\prime} \subseteq E_{k}^{n}$, если $T^{\prime} \subseteq T \cup \check{S}(T)$. Обозначим через $C(T)$ множество $E_{k}^{n} \backslash(\check{S}(T) \cup T)$ наборов, не покрытых множеством $T$.

Пусть $\delta \geqslant 0$ - действительное число. Назовём $\delta$-весом множества $T$ число $W_{\delta}(T)=|T|+\delta \cdot|C(T)|$, и пусть $p_{k}(n, \delta)=\min _{T \subseteq E_{k}^{n}} W_{\delta}(T)$. Заметим, что число $p_{k}(n, 1)$ совпадает с мощностью минимального покрытия множества $E_{k}^{n}$ (т.е. покрытия с минимальной мощностью).

Кроме того, поскольку каждый набор из $T \backslash\{\widehat{(k-1)}\}$ может быть затенён одним набором, получаем, что мощность минимального затеняющего множества на $E_{k}^{n}$ не превосходит $2 \cdot p_{k}(n, 1)$.

Пусть $\alpha \in E_{k}^{n}, \beta \in E_{k}^{m}$, где $n, m \geqslant 1$. Через $\alpha \boxminus \beta$ обозначим набор из множества $E_{k}^{n+m}$, являющийся конкатенацией набора $\alpha$ и набора $\beta$. Если $T^{\prime} \subseteq E_{k}^{n}, T^{\prime \prime} \subseteq E_{k}^{m}$, то через $T^{\prime} \boxminus T^{\prime \prime}$ обозначим множество $\left\{\alpha \boxminus \beta \mid \alpha \in T^{\prime}, \beta \in T^{\prime \prime}\right\} \subseteq E_{k}^{n+m}$.

Лемма 1. При всех натуральных $k \geqslant 2, m \geqslant 1, n \geqslant 1$ выполнено неравенство $p_{k}(n+m, 1) \leqslant k^{n} \cdot p_{k}\left(m, \frac{p_{k}(n, 1)}{k^{n}}\right)$.

Доказательство. Пусть $T^{\prime}$ - множество мощности $p_{k}(n, 1)$, покрывающее множество $E_{k}^{n}$, а $T^{\prime \prime}$ - подмножество $E_{k}^{m}$, имеющее минимальный $\delta$-вес при $\delta=\frac{p_{k}(n, 1)}{k^{n}}$. Так как множество $T^{\prime} \boxminus C\left(T^{\prime \prime}\right)$ покрывает множество $E_{k}^{n} \boxminus C\left(T^{\prime \prime}\right)$, а множество $E_{k}^{n} \sqcup T^{\prime \prime}$ покрывает множество $E_{k}^{n} \sqcup\left(E_{k}^{m} \backslash C\left(T^{\prime \prime}\right)\right)$, верно, что множество $\left(T^{\prime} \boxminus C\left(T^{\prime \prime}\right)\right) \cup\left(E_{k}^{n} \square T^{\prime \prime}\right)$ покрывает множество $E_{k}^{n+m}$.

Осталось заметить, что мощность множества $\left(T^{\prime} \bullet C\left(T^{\prime \prime}\right)\right) \cup\left(E_{k}^{n} \boxminus T^{\prime \prime}\right)$ равна

$$
\left|T^{\prime}\right| \cdot\left|C\left(T^{\prime \prime}\right)\right|+k^{n} \cdot\left|T^{\prime \prime}\right|=k^{n}\left(\left|T^{\prime \prime}\right|+\frac{\left|T^{\prime}\right|}{k^{n}}\left|C\left(T^{\prime \prime}\right)\right|\right)=k^{n} \cdot p_{k}\left(m, \frac{p_{k}(n, 1)}{k^{n}}\right) .
$$

Лемма доказана.

Лемма 2. Пусть $k \geqslant 2, u c_{k}=1+\frac{1}{k-1}$. Тогда если $\delta \geqslant \frac{c_{k}}{n}$, mо $p_{k}(n, \delta) \leqslant c_{k} \cdot \frac{k^{n}}{n} \cdot \ln \frac{e \delta n}{c_{k}}$, где е $=2,71 \ldots$ - основание натурального логарифма.

Доказательство. Построим случайное множество $T \subseteq E_{k}^{n}$, независимо выбирая в него наборы $\alpha \in E_{k}^{n}$ с вероятностью $q_{w(\alpha)}=\min \left\{1, \frac{1}{w(\alpha)+1} \cdot \ln \frac{\delta n}{c_{k}}\right\}$. Для доказательства леммы достаточно показать, что математическое ожидание $\delta$-веса построенного множества не превосходит $c_{k} \cdot \frac{k^{n}}{n} \cdot \ln \frac{e \delta n}{c_{k}}$.

Воспользуемся фактами:

1) количество наборов $\alpha$ из $E_{k}^{n}$, для которых $w(\alpha)=i$, равно $\left(\begin{array}{c}n \\ i\end{array}\right) \cdot(k-1)^{i}$;

2 ) набор $\alpha$ покрывается $w(\alpha)+1$ наборами;

3) $q_{i+1} \leqslant q_{i}$; 
4) $\alpha \in \check{S}(\beta)$ влечет $w(\alpha) \geqslant w(\beta)$;

5) $\left(1-q_{i}\right)^{i+1} \leqslant \frac{c_{k}}{\delta n}$.

Получаем:

$$
\begin{aligned}
\mathbf{E} W_{\delta}(T) & =\mathbf{E}|T|+\delta \cdot \mathbf{E}|C(T)|= \\
& =\sum_{\alpha \in E_{k}^{n}} q_{w(\alpha)}+\delta \cdot \sum_{\alpha \in E_{k}^{n}} \mathbf{P}(\alpha \in C(T)) \leqslant \\
& \leqslant \ln \frac{\delta n}{c_{k}} \cdot \sum_{i=0}^{n}\left(\begin{array}{c}
n \\
i
\end{array}\right)(k-1)^{i} \frac{1}{i+1}+\delta \cdot \sum_{i=0}^{n}\left(\begin{array}{c}
n \\
i
\end{array}\right) \cdot(k-1)^{i} \cdot\left(1-q_{i}\right)^{i+1} \leqslant \\
& \leqslant \ln \frac{\delta n}{c_{k}} \cdot\left(1+\frac{1}{k-1}\right) \cdot \frac{k^{n}}{n+1}+\delta \cdot \frac{c_{k}}{\delta n} \cdot \sum_{i=0}^{n}\left(\begin{array}{c}
n \\
i
\end{array}\right) \cdot(k-1)^{i} \leqslant \\
& \leqslant c_{k} \cdot \frac{k^{n}}{n}\left(\ln \frac{\delta n}{c_{k}}+1\right) .
\end{aligned}
$$

Лемма доказана.

При каждом натуральном $k, k \geqslant 2$, положим $c_{k}^{\prime}=2 c_{k} \cdot\left(-W_{-1}\left(-\frac{1}{2 e}\right)\right)$, где $W_{-1}\left(-\frac{1}{2 e}\right) \approx-2.67835$ - значение второй ветви $W$-функции Ламберта [11]: $W_{-1}\left(-\frac{1}{2 e}\right) \cdot e^{W_{-1}\left(-\frac{1}{2 e}\right)}=-\frac{1}{2 e}$. Отметим, что $c_{k}^{\prime}$ является решением уравнения $c_{k}^{\prime}=2 c_{k} \ln \frac{e c_{k}^{\prime}}{c_{k}}$.

Лемма 3. Пусть $k \geqslant 2 u r$ - целое неотрицательное число. Тогда выполнено неравенство $p_{k}\left(2^{r}, 1\right) \leqslant \frac{c_{k}^{\prime} \cdot k^{2^{r}}}{2^{r}}$.

Доказательство. Докажем утверждение леммы индукцией по $r$. При $r=0$ утверждение выполнено, поскольку $p_{k}\left(2^{0}, 1\right)=\left\lceil\frac{k}{2}\right\rceil \leqslant c_{k}^{\prime} k$. Теперь предположим, что $p_{k}\left(2^{r}, 1\right) \leqslant \frac{c_{k}^{\prime} \cdot k^{2^{r}}}{2^{r}}$, и оценим $p_{k}\left(2^{r+1}, 1\right)$. Используя лемму 1 , полагая в ней $m=n=2^{r}$, а затем лемму 2 при $\delta=\frac{c_{k}^{\prime}}{2^{r}} \geqslant \frac{c_{k}}{2^{r}}$ и $n=2^{r}$, получаем

$$
\begin{aligned}
p_{k}\left(2^{r+1}, 1\right) & \leqslant k^{2^{r}} \cdot p_{k}\left(2^{r}, \frac{p_{k}\left(2^{r}, 1\right)}{k^{2^{r}}}\right) \leqslant \\
& \leqslant k^{2^{r}} \cdot p_{k}\left(2^{r}, \frac{c_{k}^{\prime}}{2^{r}}\right) \leqslant \\
& \leqslant \frac{c_{k} \cdot k^{2^{r+1}}}{2^{r}} \cdot \ln \frac{e c_{k}^{\prime}}{c_{k}}=\frac{c_{k}^{\prime} \cdot k^{2^{r+1}}}{2^{r+1}} .
\end{aligned}
$$

Лемма доказана.

Теорема 2. При каждом натуральном $k \geqslant 2$ и при каждом иелом $n \geqslant 0$ существует множество мощности $\frac{2 c_{k}^{\prime} \cdot k^{n}}{n}$, покрывающее множество $E_{k}^{n}$.

Доказательство. Докажем, что $p_{k}(n, 1) \leqslant \frac{2 c_{k}^{\prime} \cdot k^{n}}{n}$. Выберем такое целое неотрицательное число $r$, что $2^{r} \leqslant n<2^{r+1}$. Заметим, что если $T$ покрывает множество $E_{k}^{2^{r}}$, то $T \boxminus E_{k}^{n-2^{r}}$ покрывает множество $E_{k}^{n}$, и поэтому

$$
p_{k}(n, 1) \leqslant p_{k}\left(2^{r}, 1\right) \cdot k^{n-2^{r}} \leqslant \frac{c_{k}^{\prime} \cdot k^{n}}{2^{r}} \leqslant \frac{2 c_{k}^{\prime} \cdot k^{n}}{n} .
$$

Теорема доказана.

Теорема 3. Если $k$ - простое число, то $L_{k}^{p . n . f .}(n)=O\left(\frac{k^{n}}{n}\right)$.

Теорема 3 следует из теорем 1 и 2 .

Применяя нижнюю мощностную оценку для $L_{k}^{p . n . f .}(n)$ из [8], получаем окончательный результат.

Теорема 4. Если $k$ - простое число, то $L_{k}^{p . n . f \cdot}(n)=\Theta\left(\frac{k^{n}}{n}\right)$. 


\section{Список литературы}

1. Угрюмов Е.П., Цифровая схемотехника, СПб.: БХВ-Петербург, 2004.

2. Astola J.T., Stankovich R.S., Fundamentals of Switching Theory and Logic Design, Springer, Dordrechht, The Netherlands, 2006.

3. Sasao T., Besslich P., "On the complexity of mod-2 sum PLA's", IEEE Trans. Comput., 39:2 (1990), 262-266.

4. Супрун В.П., "Сложность булевых функций в классе канонических поляризованных полиномов", Дискретная математика, 5:2 (1993), 111-115.

5. Перязев Н. А., "Сложность булевых функций в классе полиномиальных поляризованных форм", Алгебра и логика, 34:3 (1995), 323-326.

6. Селезнева С.Н., "О сложности представления функций многозначных логик поляризованными полиномами", Дискретная математика, 14:2 (2002), 48-53.

7. Кириченко К. Д., "Верхняя оценка сложности полиномиальных нормальных форм булевых функций”, Дискретная математика, 17:3 (2005), 80-88.

8. Селезнева С.Н., Дайняк А. Б., "О сложности обобщенных полиномов $k$-значных функций”, Вестник Московского университета. Серия 15. Вычислительная математика и кибернетика, 2008, № 3, 34-39.

9. Яблонский С. В., Введение в дискретную математику, М.: Высшая школа, 2001.

10. Cooper J. N., Ellis R.B., Kahng A.B., "Asymmetric binary covering codes", J. Comb. Theory, Ser. A, 100:2 (2002), 232-249.

11. Corless R. M., Gonnet G.H., Hare D. E., Jeffrey D. J., Knuth D. E., "On the LambertW function. Adv. Comput. Math.", 5:1 (1996), 329-359. 